\title{
CENTRAL AND NONCENTRAL COMPONENTS OF THE PREEDOM-WILDENTHAL INTERACTION
}

\author{
G.A. TIMMER, R. VAN DER HEYDEN and B.C. METSCH \\ Fysisch Laboratorium, Rijksuniversiteit Utrecht, The Netherlands \\ and \\ K. KLINGENBECK \\ Institut für Theoretische Physik, Universität Erlangen-Nürnberg, Erlangen, West Germany
}

Received 17 October 1978

\begin{abstract}
The influence of the various components of the Preedom-Wildenthal interaction - viz., central, tensor, spin-orbit (LS) and antisymmetric spin-orbit (A $L S$ ) - on properties of $A=20,21$ nuclei is investigated. The effects of the $L S$ and A $L S$ components on the one hand and of the central and tensor components on the other hand are found to be very similar. It turns out to be possible to remove the A $L S$ component in favour of an enhanced $L S$ component without spoiling the agreement with experiment.
\end{abstract}

Following the techniques described in refs. [1-3] one can write a given effective two-body interaction as a sum of central (C), tensor (T), spin-orbit $(L S)$ and antisymmetric spin-orbit (ALS) components. In order to discern the influence of the various components, the interaction $V=\Sigma V_{i}$ is written here as $\Sigma$ $\lambda_{i} V_{i}$ with $\lambda_{i}$ denoting strength parameters that are to be varied $(i=\mathrm{C}, \mathrm{T}, L S, \mathrm{~A} L S)$. In the calculations to be described below the Preedom-Wildenthal (PW) interaction [4] was employed, i.e. $V_{\mathrm{PW}}=\Sigma V_{i}$. No separate strength parameters for the $T=0$ and $T=1$ channels were considered.

In a first step the energy spectra and electromagnetic properties of $A=20$ and $A=21$ nuclei were calculated as a function of the strength parameters. A rather detailed account along these lines has been given in ref. [2] for the case of ${ }^{24} \mathrm{Mg}$. In ref. [2] it was stressed that a large enhancement of the $\mathrm{A} L S$ strength $\left(\lambda_{\mathrm{A} L S} \approx 5\right)$ of the Kuo-Brown interaction in a SU(3) truncated model space led to a marked improvement in the description of ${ }^{24} \mathrm{Mg}$. Our calculation on $A=20$ and $A=21$ nuclei led to a different picture. The principal result was a remarkable coherence in the effects of varying $\lambda_{L S}$ or $\lambda_{\mathrm{A} L S}$ on the one hand and $\lambda_{\mathrm{C}}$ or $\lambda_{\mathrm{T}}$ on the other hand. In fig. 1 this is illustrated for magnetic moments. The values in fig. 1 were obtained upon variation of one strength parameter at a time, while the others were fixed at unity. With relatively few exceptions the same clear trends persisted for other quantities (E2 and M1 transition rates, quadrupole moments, wave function overlaps etc.) as well. Only for energies, $\mathrm{C}$ and $\mathrm{T}$ showed an opposite behaviour, see fig. 2. The low-lying states of ${ }^{20} \mathrm{Ne}$ are of a rather pure $S=0$ nature, henceforth preventing a sizable first-order effect of the noncentral components. Nevertheless, the trends mentioned before emerged in this case also.

The coherence of the $L S$ and ALS effects suggests that it might be possible to remove the A $L S$ component in the PW interaction in favour of an enhanced $L S$ component. Although there have been given some explanations [2,3] for the presence of an $\mathrm{A} L S$ term, it has not yet been possible to justify the large contributions of the ALS term to an effective interaction. Hence, in our opinion it is worthwhile to postpone as long as possible the introduction of such a term which is virtually absent in the free nucleon-nucleon interaction. 

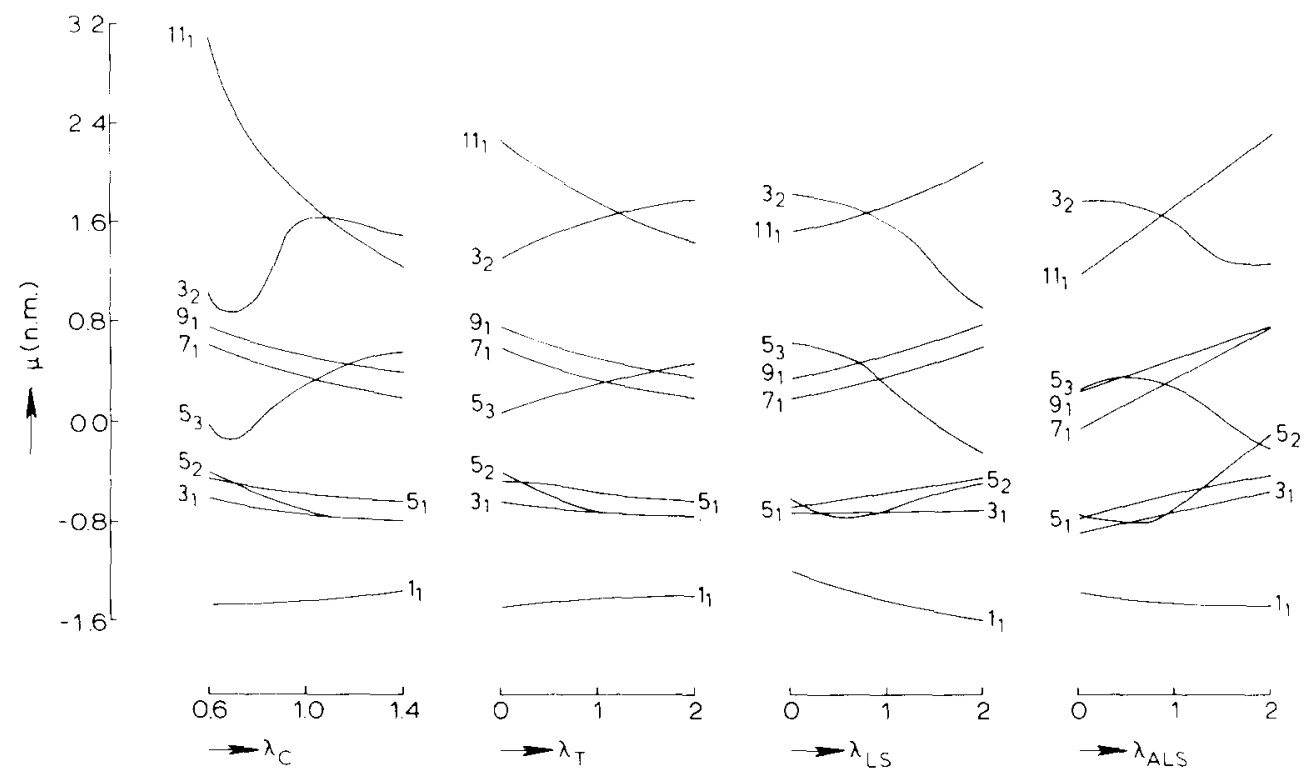

Fig. 1. Magnetic moments of levels in ${ }^{21} \mathrm{Ne}$ calculated from wave functions belonging to hamiltonians $V_{\mathrm{PW}}+\left(\lambda_{i}-1\right) V_{i}$ where $i=\mathrm{C}, \mathrm{T}, L S, \mathrm{~A} L S$ for the columns $1-4$, respectively. Bare nucleon $g$-factors were employed.

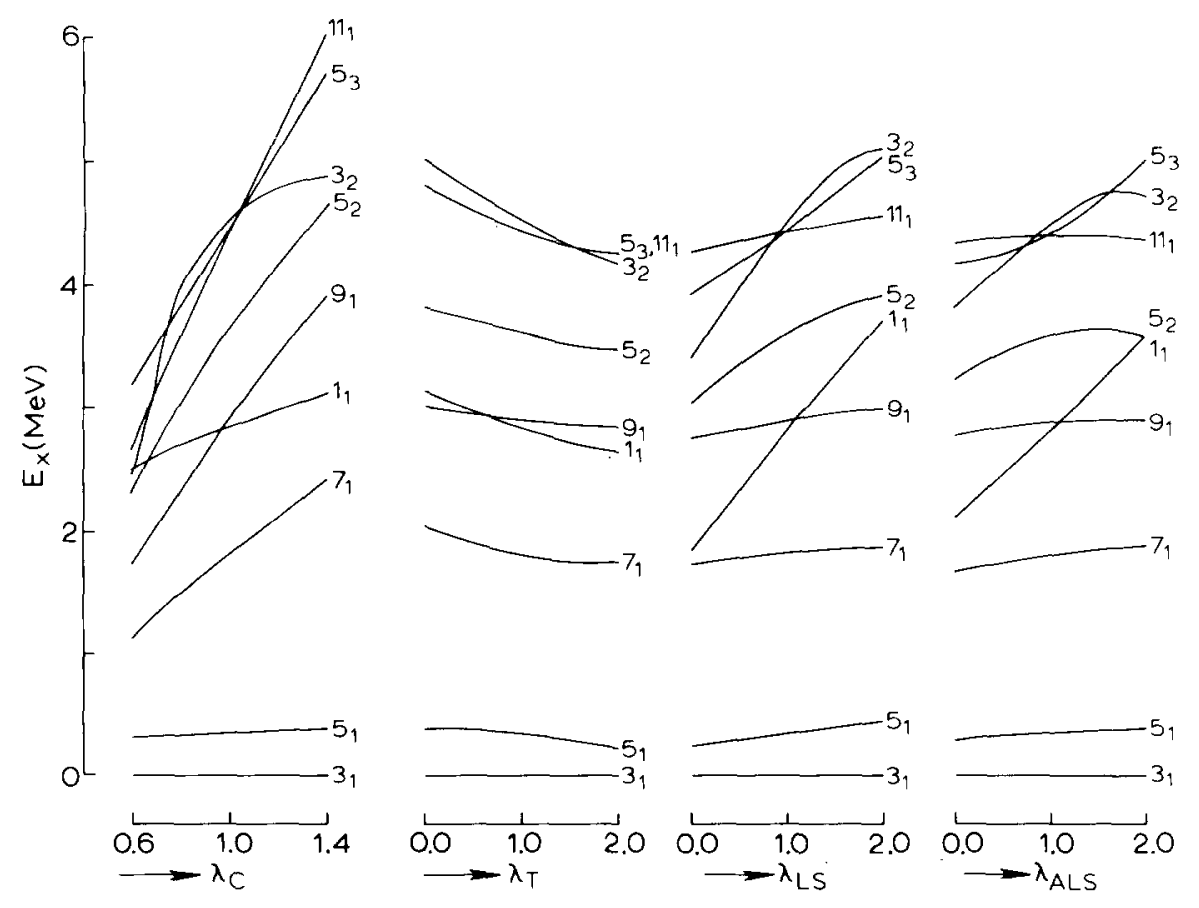

Fig. 2. Excitation energies of levels in $A=21, T=1 / 2$ nuclei for the same hamiltonians as used in fig. 1 . 


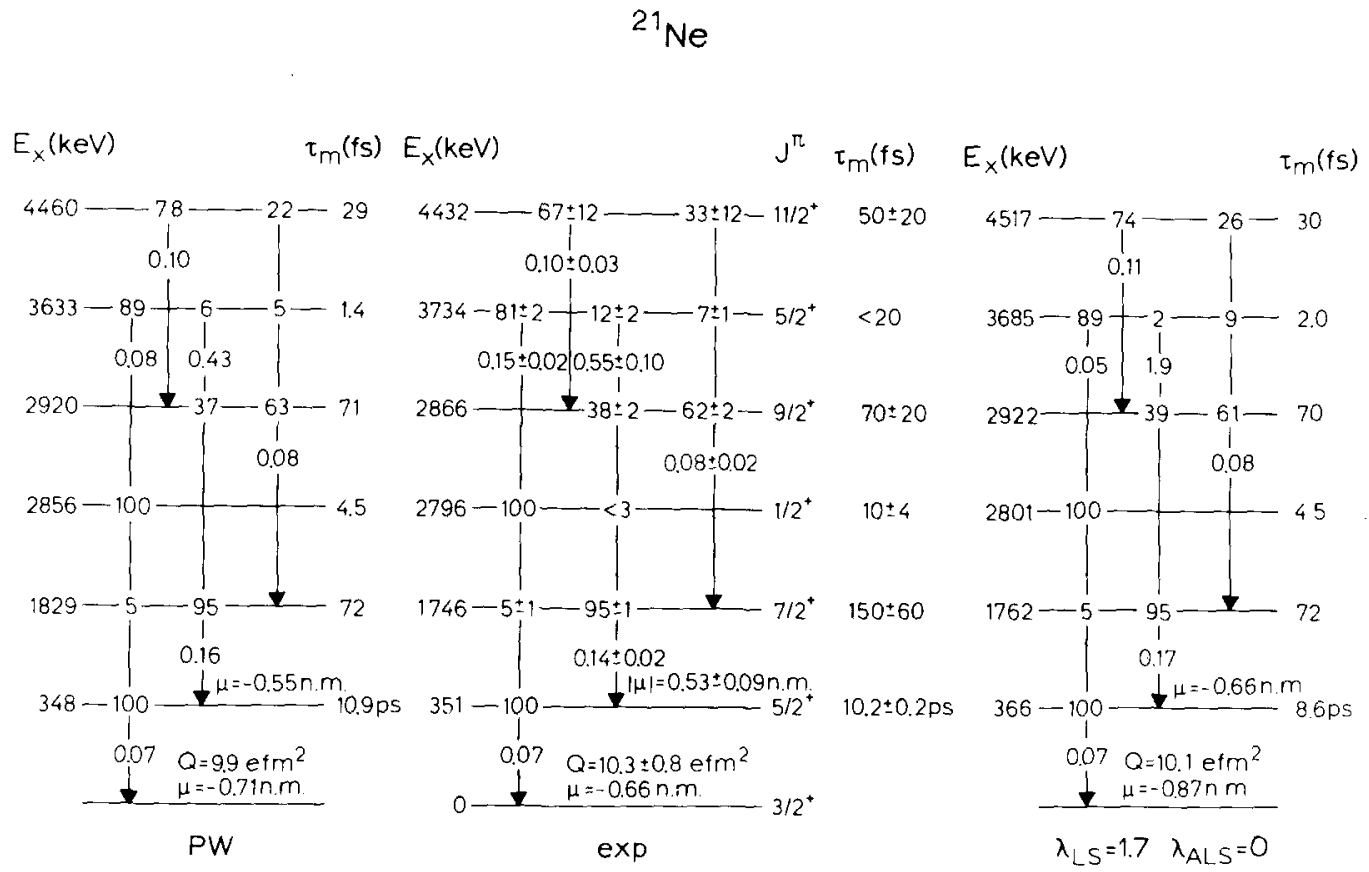

Fig. 3. Decay scheme of ${ }^{21} \mathrm{Ne}$. Experimental data were taken from ref. [5]. The first column refers to the Preedom-Wildenthal interaction. Differences with results presented in ref. [4] are due to a slightly different set of single-particle energies. For the third column see the text.

In fig. 3 the result of a calculation with $\lambda_{C}=\lambda_{T}=1$, $\lambda_{\mathrm{A} L S}=0$ and $\lambda_{L S}=1.7$ is displayed. The value for $\lambda_{L S}$ was obtained by some trial and error in such a way as to reproduce the experimental excitation energy of the $1 / 2_{1}^{+}$level; the position of the other low-lying levels is less strongly dependent on the strength parameters. The agreement with experiment is of the same quality as for the PW interaction. Only for the magnetic moment of the ground state the compensating effect of the $L S$ term is too small; see also fig. 1. Preliminary calculations on $A=22$ nuclei confirm the conclusions drawn here.

A more complete account of this work will be published elsewhere.
One of the authors (G.A.T.) wants to express his gratitude to Professor M.G. Huber for the hospitality encountered in Erlangen where this work started.

\section{References}

[1] M.W. Kirson, Phys. Lett. 47B (1973) 110.

[2] H. Feldmeier, P. Manakos and T. Wolff, Z. Phys. 258 (1973) 81

[3] K. Klingenbeck, W. Knüpfer, M.G. Huber and P.W.M. Glaudemans, Phys. Rev. C15 (1977) 1483.

[4] B.M. Preedom and B.H. Wildenthal, Phys. Rev. C6 (1972) 1633.

[5] P.M. Endt and C. van der Leun, $1978 A=21-24$ compilation, to be published. 